\title{
SCIATICA
}

\section{AN ANATOMICAL AND MECHANICAL STUDY OF THE LUMBOSACRAL REGION}

\author{
BY
}

\section{NORMAN CAPENER}

\section{Princess Elizabeth Orthopaedic Hospital, Exeter}

Great interest is being shown in sciatica at the present time. It is a common disturbance of obscure etiology. Its association with rheumatism and trauma renders it a severe economic problem, in war as in peace. An advance in the understanding of some forms of sciatica has been made in the recognition of the important part played by intervertebral disc lesions (Alajouanine and Petit-Dutaillis. 1930; Mixter and Barr, 1934; Peet and Echols, 1934). A certain amount of clinical research is being conducted upon special groups of cases. It is probable that other and more extensive investigations will be embarked upon as soon as circumstances permit. Therefore I have felt that the time is appropriate for the publication of certain researches into the anatomical and mechanical background of the problem.* Very little originality is claimed for the greater part of the work. Material (both clinical and radiological) has been studied and is here presented in the hope that it may prove a guide for investigators. Written as it is from the orthopaedic point of view, it is natural that the mechanical factors should be stressed rather than the pathological. I have tried to avoid glaring excursions into theoretic considerations, but it is impossible to avoid them altogether.

\section{The Normal Lumbar Curve}

The lumbar hollow is a human characteristic which babies acquire in the first year of life (Fig. 1). It is dependent upon extension of the thighs, which

* Based on a Hunterian Lecture, "The Lumbo-sacral Joint: a Discussion upon Spondylolisthesis, Scoliosis, and Sciatica," Royal College of Surgeons; Jan. 22, 1941. before birth have been held flexed upon the abdomen. After birth some " fixed" flexion of the hips remains. Extension force applied to these joints in small babies is communicated to the lumbar spine and a lordotic concavity is developed. Willis (1932) has suggested that the deleterious effects of such force upon the lumbar spine are aggravated in civilized people by " the efficient bathing, diapering, and swaddling attentions of the modern nurse." Certainly lordosis and protuberant abdomen are normal features in infants who are learning to walk; the relation of this in some children to lack of hip extension is to be remembered. Later, as true extension of the hip-joints occurs, there is shortening of the ham-string muscles, the influence of which, by tilting the pelvis backwards, is to reduce the lumbar lordosis. Any circumstance which unduly increases ham-string tension will restore the prenatal type of lumbar convexity backwards (Fig. 1, D). This is by no means an unusual circumstance, for it is to be found in any person who chooses to sit upright with his knees extended; or, with the knees extended, to pick up something from the ground. As we shall see later, the latter position is fraught with danger for those prone to sciatica, and the former is one all too frequently indulged in by those who spend too much time in bed. It is more than of passing interest that while the prenatal condition of the foetal lumbar spine is one of backward convexity, so, as Lambrinudi (1934) observed, is that of its mother's; which is not lordotic as so commonly thought. The condition of the gravid woman's

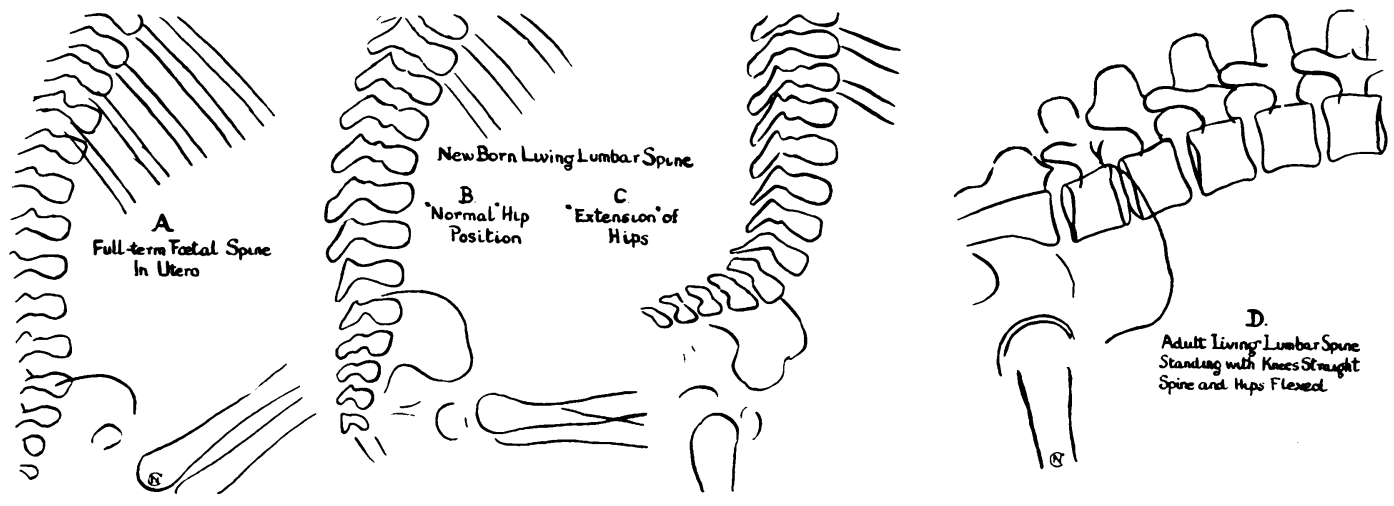

Fig. 1. The Lumbosacral Paradox. Vulnerability in infancy by deviation from prenatal curvature-i.e. by extension force. Vulnerability in adult life by return to prenatal type of curvature-i.e. by flexion force. 
lumbar spine from the sixth month onwards is favourable for the development of sciatica.

\section{Lumbar Spine as a Unit}

The Question of Rotation.-Much of anatomical description in textbooks is directed to the varying features of individual segments, their shape, attachments and articulation. Too little attention, however, is paid to the spine as a whole, or to its regions as units of the whole. The spine is a concatenation and behaves like a chain. Movement of one segment involves movement of the whole. The periodicity of scoliosis, which so closely resembles a phase of vibration in a monochord, has its counterpart in the twists and curves of unstable pillars and towers. This is too big a subject to go into here, but if we do realize the obvious idea of the chain, it will be much easier to understand the question of whether rotation movements occur in the lumbar spine. Many writers still quote old textbook descriptions denying it, and J. E. Frazer still repeats that the articular facets of the lumbar vertebrae are so placed as to prevent rotation, which is only considered as being around a vertical axis situated in the centre of the vertebral bodies (Fig. 4). If, however, we disregard the segmental idea, there is no reason at all why the axis of rotation should not be found anywhere, even in mid-air. Certainly, from a mechanical viewpoint, one would expect it to lie on the concave side of the normal curve (as it does in the thoracic region). To everyone familiar with the problems of scoliosis it is obvious that a considerable degree of rotation does occur in the lumbar region. If the axis for this movement is regarded as being posterior to the spinal canal, then it is quite easy to see how the arrangement of the lumbar articular facts readily permits it. The range need be less than eight degrees between each segment to permit a total of forty-five degrees between the chest and the pelvis, which is probably as much as a normally supple person requires, and is more than he could produce in the thoracic region, which, relatively, has far less rotation because of the need for protection of its viscera. Owing to the more coronal arrangement of the lumbo-sacral facets it is probable that a larger degree of rotation is possible here than in the rest of the lumbar region. There can be no doubt about this matter of rotation for another reason-because it is a physical necessity in any lateral flexion. Bending sideways any structure like the lumbar spine, which has a pre-existing forward convexity, must be accompanied by torsion of the whole region (or rotation between individual segments).

The posterior neural arches and spinous processes are of greater mechanical importance than has generally been realized. Under the heading of " weight transmission " I shall have more to say, but here one should remark on an interesting phenomenon in the movement of hyper-extension: not only does weight tend to be transferred to the articular and spinous processes, but individual vertebrae tend to slide backwards on the one below. It is more readily detected in the upper lumbar region, and was well shown in the extreme example of lumbar hyperextension in a contorsionist whose radiograph was described by Lockhart and reproduced in the last edition of Cunningham's Textbook of Anatomy. I have demonstrated the same point in radiographs of normal spines (Fig. 2), and (Capener, 1932) in a remarkable case of spinal tuberculosis

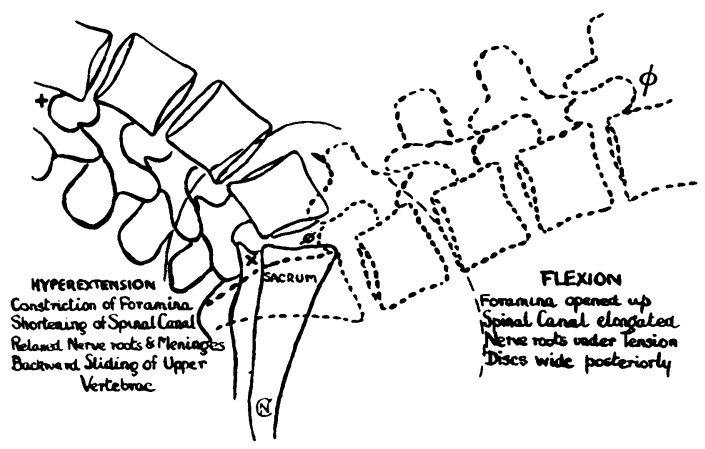

FIG. 2. Two views of the same spine in standing position. In the original films the distance between the same points marked $X$ in extension and $\varnothing$ in flexion were $5 \frac{5}{8}$ in. and $7 \frac{5}{8}$ in. respectively.

associated with spondylolisthesis (Fig. 5). In the upper lumbar spine it may become a fixed condition and is then known as retrospondylolisthesis; being often due to post-traumatic degeneration of the intervertebral disc, or osteoarthritis.

\section{Fourth Lumbar Vertebra}

The importance of this vertebra in the present discussion is that it is the "spear head of attack" by superimposed body weight upon the mechanical weaknesses of the fifth lumbar vertebra. It is often the last vertebra of completely lumbar type. From the intervertebral disc beneath it there occurs posterior herniation with almost equal frequency as from the lumbo-sacral disc. In the past, prominence has been given to the various forms of true congenital anomaly of the fifth lumbar vertebra, such as complete or partial sacralization. Generally, attention has been paid to the aberrant masses which project laterally from one or other transverse process and articulate more or less completely with the lateral mass of the sacrum. Probably osteoarthritic changes can occur at these additional joints, but what tends to be overlooked is the consequent altered mechanical conditions in the joints between the fourth and fifth lumbar vertebrae. With the greater fixation of the fifth, the stresses to which the fourth is exposed without doubt explain such a circumstance as the greater liability then for the fourth to show spondylolytic changes, and of its intervertebral disc to suffer from trauma.

\section{Intervertebral Discs}

From a study of recent literature, it is obvious that these structures are of considerable significance in any discussion of sciatica. The best work upon the normal and morbid anatomy is that by Schmorl, made readily available to English readers by Beadle 
(1931). I shall concentrate upon mechanical features.

Each disc is composed of two elements: (1) an annulus fibrosus, which is thinner posteriorly, and encloses (2) the nucleus pulposus, a somewhat spherical structure under tension, which is partly given to it by the encasing fibres of the annulus. The nucleus lies behind the centre of the disc, its position being recognized in a lateral radiograph by the slight hollow above and below in the outlines of the contiguous vertebral bodies. At the periphery each disc is bound down to the intervertebral ligaments, especially in front to the anterior common ligament, and to the slighter posterior common ligament behind. During movements of the spine it is apparent that change of shape must occur in each disc. In flexion and extension (Fig. 2) compression of one edge is accompanied by expansion of the opposite side. In lateral flexion and rotation (Fig. 3), and one cannot occur without the other,

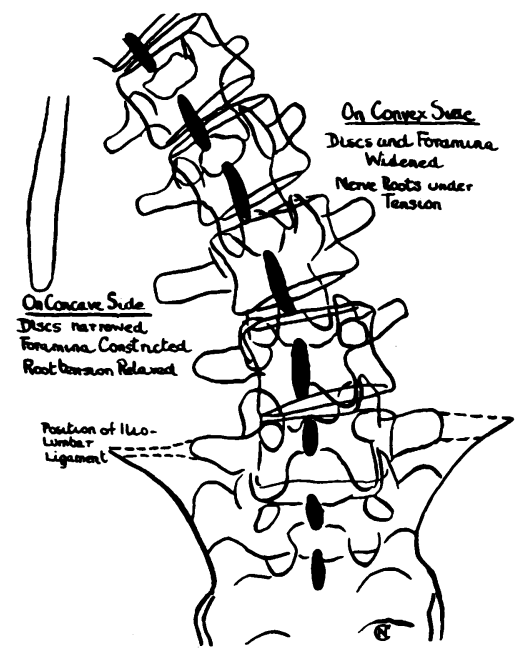

Fig. 3. Another standing spine showing lateral flexion and rotation.

considerable distortion must occur. In hyperextension a special sort of shearing effect must be present to allow of the backward sliding of the upper vertebrae already noted. A study of radiographs of the same spine, in different phases of movement, suggests that the nucleus pulposus is the pivot around which the more simple movements occurin fact, rather like a see-saw. Such radiographs also can tell us something about the internal stresses of the vertebral bodies (Fig. 5). Where a portion of the disc is narrowed the parts of the vertebra above and below must also be in a state of compression, and when the disc is widened so the vertebral substance above and below must be in relative tension.

Let us consider posterior protrusion into the spinal canal of annulus or nuclear substance. One should realize that the annulus is weakest at this point, and that in flexion of the lumbar spine the internal stresses within the disc are relatively tensile or negative posteriorly, and predominantly compressive or positive anteriorly. The whole tendency,

therefore, in forced lumbar flexion is for the nuclear substance to be expressed backwards like a " pea out of its pod." Any movement in the direction of flexion of the lumbar spine with the hips fixed in relative extension, as when the knees are straight, will, for reasons already mentioned, convey a greater flexion force to this part of the spine-a

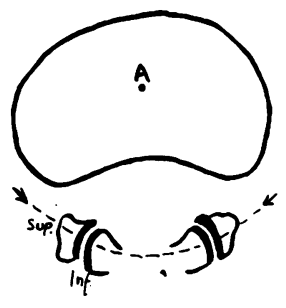

A.

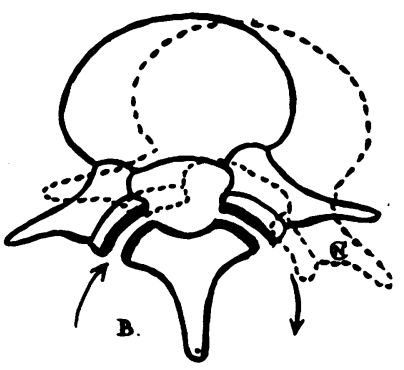

FIG. 4. Lumbar Rotation. A.-After Fraser who states there is none. B.-Author's interpretation (diagrammatic).

force which will be increased from above should the individual be lifting a weight. So important is the danger of this position regarded by certain American industries that placards are exhibited in workshops discouraging it and advising workmen to bend their knees and so "get under their weights." For a similar reason the habitual position of the invalid

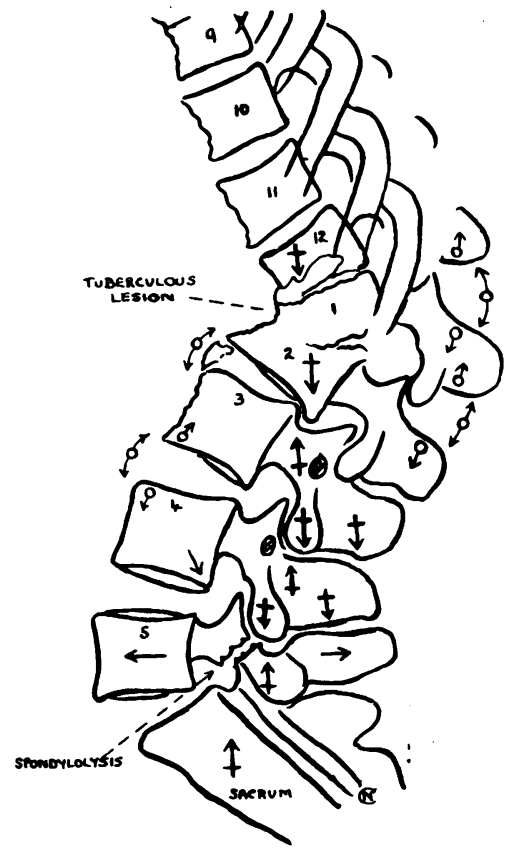

FIG. 5.-A demonstration of stresses in a pathological spine.

$\mathrm{O}=$ tension. $\quad+=$ compression. Arrows indicate direction of force or movement.

who sits in bed with knees straight is also dangerous. An important point, noted by Schmorl, is the frequency with which disc protrusions within the spinal canal are found at autopsy; it seems that this is consistent with freedom from sciatic pain Whether a retropulsed nucleus can slip partially backwards 
again is open to doubt. It certainly would seem unlikely that it could return completely. The idea of spontaneous reduction is attractive to the surgeon who at operation finds nothing when lipiodol radiography has been clearly positive. In such circumstances it should be recognized that the influence of weight-bearing is removed and that in recumbency there is a natural tendency for the intervertebral discs to resume a normal shape. It would be interesting to know if the amount of retropulsion is a variable phenomenon in any particular case.

\section{The Meninges and Intervertebral Foramina}

In the spinal canal the meningeal tube is separated from its bony and ligamentous surroundings by an appreciable layer of extradural fatty areolar tissue which permits easy movement. The amount of this movement requires further attention, for it is surprising to most people.

If one compares lateral radiographs of the lumbar spine in full flexion with those of the same spine in hyperextension, the disparity in length is remarkable. In the illustrations (Fig. 2) showing this, measurements were taken between the two same points in the canal, and the elongation in full flexion is found to be at least one-third of the shorter length in hyperextension. Obviously, in flexion the meninges and cauda equina are under relative tension. It is the same position of danger for intervertebral discs and nuclei, so that the retropulsive tendencies of them are met by nerve tissues on the stretch. Conversely, in hyperextension relaxed meninges and nerves go with closed-up posterior disc spaces. While discussing the effect of lumbar movement and posture upon the intraspinal soft tissues one should mention changes occurring within the intervertebral foramina. First of all, there is interest in the feature to which attention was drawn by Danforth and Wilson (1925). Throughout the lumbar series these foramina become progressively smaller from above downwards to the lumbo-sacral interspace. This, which transmits the largest nerve root of them all, has the smallest available space. We must realize that each foramen also contains meningeal blood vessels and a prolongation of dura which eventually merges with the perineural sheath. Furthermore, each nerve root, which has emerged at an angle from beneath the pedicle above, has a variable degree of prolongation of arachnoid with cerebro-spinal fluid (Capener, 1937) (Fig. 6). This extension of the theca, particularly on the lower lumbar nerve roots, is generally not appreciated.

In full flexion of the lumbar spine the foramina are enlarged to their maximum, in hyperextension they are smallest (Fig. 2). The vulnerability of structures within them is greatest in hyperextension, which is the reverse to the dangers to structures within the spinal canal. In these altered relations we have, I believe, some explanation for the conflicting signs that many patients present. The diagram shows this graphically (Chart 1). In some

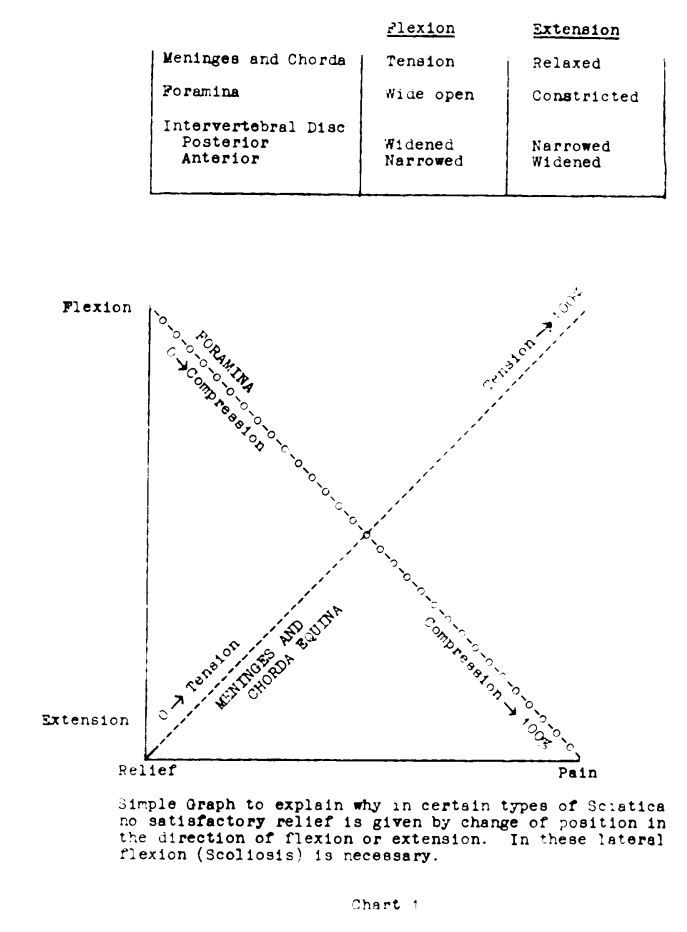

patients relief from pain is given by hyperextension; in others, by flexion. In the former, relief may be due to relaxation of the cauda equina and meningeal tension or of nerve-root kink: while in flexion relief may result because there is greater room for the foraminal contents. On the contrary, flexion
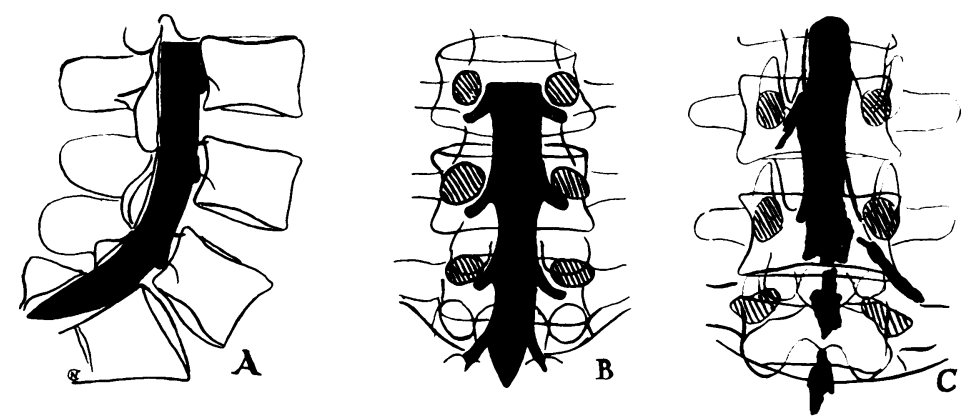

FIG. 6. Relation of nerve roots, pedicles and intervertebral foramina. A and B.-Autopsy specimen in which theca has been filled with lipiodol. C.-Radiograph taken in course of investigation of a case of nuclear retropulsion. 
may increase disc and nuclear retropulsion, while by hyperextension one may aggravate pain by constricting foraminal contents. Lateral flexion will have a similar effect on the concave side, and rotation will increase nerve-root tension on one side. The constriction of intervertebral foraminal contents is, of course, of more significance should the nerve root be oedematous, or if the size of the foramen is further reduced by lateral disc protrusion, osteoarthritic outgrowth, and so forth. Here it should be noted that herniation of disc substance into a foramen is not seen in opaque myelography. Some of the most distressing forms of sciatica are in individuals who get relief by no alteration in position, and in whom every movement is agonizing. It will readily be appreciated from the above discussion how movement giving relief from one element of the painful condition is vitiated by the aggravation of something else. Nevertheless, posture does sometimes produce the most astonishing relief. When dealing with muscular function later on I shall have more to say on this; but here one should remark upon the fact that hyperextension occasionally is very helpful. It suggests that a nerve root, caught upon the summit of a bulging intervertebral disc, may be dislodged into the neighbouring valley by the backward sliding tendency of the upper vertebra, at a time when the chorda equina and meningeal tension is eased. Sudden jerks may do the same thing.

A phenomenon which illustrates the complexities of spinal mechanics is the peculiar scoliotic displacement of the trunk which is almost invariably associated with sciatica. Most frequently such displacement is to the opposite side (contralateral) and probably is to be interpreted as an attempt to relieve intervertebral compression; when the curvature is towards the same side (ipsi- or homo- lateral) it is probably nerve-root tension which the body is trying to avoid. Another element of deformity usually present is elimination of lordosis; such flattening of the lumbar hollow may be so severe as to constitute a lumbar kyphos. These deformities which are fixed by muscle spasm are comparable to the hamstring spasm which fixes a deranged knee in flexion. A rarer anomaly is an alternating type of sciatic scoliosis (ischiatica alternans) in which the patient may vary the side of his displacement, sometimes voluntarily; at one moment ipsilateral, another time contralateral; but never can he hold the spine straight. Closer analysis of such a case reveals that the curves are not mirror images of each other. In one case reported by the writer (Capener, 1933) it was found that ipsilateral scoliosis had its apex at a higher level in the lumbar spine than the contralateral curvature.

I have often wondered whether herniation of the arachnoid farther into an intervertebral foramen occurs; possibly through local defect in its coverings or from frequent sudden rises of intrathecal cerebrospinal pressure. In one case of sciatica (Fig. 6, C), radiographed with lipiodol, I did find an unusually long prolongation around a nerve root beyond the confines of the intervertebral foramen. This, of course, need not be the explanation of the commonly observed remark of patients that sciatic pain is increased by sneezing or coughing. The bombardment of an oedematous nerve root by a sudden wave of increased intrathecal pressure is sufficient to account for it. Compression of the jugular veins will act in the same way: for example, with the patient sitting comfortably and the knee of the affected side extended to a point just short of that necessary to cause sharp sciatic pain, compress the jugulars-with intraspinal lesions the patient will probably experience sharp pain in the affected sciatic distribution. Even more significant is it when the test is carried out on the unaffected leg and pain is produced on the affected side. In such tests it must be remembered that straight leg raising (Laseague's test) necessarily causes elimination of the normal lumbar curve and produces all that goes with lumbar flexion. Laseague's test on the unaffected limb, if it gives pain on the affected side, is much more significant than when performed on the affected side alone. It does then indicate that pain is being produced by spinal movement, and not by stretching the affected sciatic nerve trunk.

\section{The Ligaments}

Two ligamentous structures in this region call for particular comment. The first is the ligamentum flavum, which has come into prominence recently because of the more frequent operations for sciatica. This "yellow" ligament is an interesting structure because, in a histological sense, it is composed of almost pure elastic tissue fibres and because a condition of " hypertrophy" has been described. It bridges the gap between each neural arch posteriorly; it thus completes the protection of the intraspinal tissues, and because of its density and elasticity acts like the flexible sheath to a motor brake-rod or carburettor control. At operation it is not infrequently found to be thickened, and appears to constrict the deeper tissues. Dockerty and Love (1940) have shown, however, that, rather than hypertrophy, the condition is the result of degeneration, and the thickening is due to invasion and replacement of elastic tissue by fibrous tissues. Probably it is secondary to local irritative changes-e.g. osteoarthritis or nuclear retropulsion. It is a convenient condition on which to pin a diagnosis when laminectomy is otherwise negative; but we deceive ourselves if we think that this is the real cause of the patient's trouble.

The ilio-lumbar ligaments provide an important means of anchoring the fifth lumbar vertebra. This vertebra has potentialities for considerable rotation, and is exposed to an amount of shearing stress not found elsewhere in the spine. Extending from the transverse processes (which are placed relatively more anteriorly) these ligaments (Fig. 3) are attached, at each side, to the crest of the ilium; are well placed for the control of rotation, and together provide a sling restraining forward movement of the last lumbar vertebra. That this is so is shown 
decisively by some patients with spondylolisthesis, where the ligaments become calcified, as so often happens when ligamentous bands are exposed to long-standing tension of a pathological degree (e.g. the interspinous ligaments in tuberculous kyphosis). It requires little stretch of the imagination to envisage lesser degrees of ligamentous fibrosis as the result of trauma, such as, for example, occurs elsewhere in occupational lesions. It is worth noting, in passing, that from this ligament arises some of the fibres of the quadratus lumborum muscle.

\section{Weight Transmission}

So far I have discussed the vulnerability of the lumbo-sacral region mainly from the aspect of dynamics. There are, however, questions of statics which are no less important. Let us start first with the lumbo-sacral angle, which has been variously described by different authors. Most frequently it is used to denote the angle formed between the anterior surfaces of the last lumbar and first sacral bodies. Considered in terms of statics, the angle described by Whitman is more significant. According to him the lumbo-sacral angle is that between the upper surface of the sacrum and the horizontal. It matters little whether this angle is measured thus or in relation to the vertical. To avoid confusion I will use the term "lumbo-sacral inclination," which from a mechanical viewpoint is the angle to the horizontal of a plane midway through the lumbosacral intervertebral disc. There is no record that lumbo-sacral angles have ever been measured in living erect human beings: one suspects that measurements have been done on cadavers or in recumbency-both fallacious. I have radiographed the lumbo-sacral inclination in a number of standing individuals and have found the angle to be about 25-30 degrees (Fig. 7B). In the habitual lordotic person the angle is greater; in an individual with short hamstrings it is less. The angle is of course very variable according to posture. When the spine is hyperextended, or when the individual sits with knees extended, the inclination becomes almost horizontal; as it also does with Goldthwaite's backflattening exercise. When standing with the spine and hips flexed, the lumbo-sacral intervertebral plane tilts forward to the vertical and beyond. In the joint between the fourth and fifth lumbar vertebra the plane of the intervertebral disc, except in lordotic people, is almost horizontal. The importance of the lumbo-sacral inclination is in the shearing stress exerted upon the intervertebral disc, and the consequent tendency for the whole lumbar spine to slide forwards off the sacrum. That this sliding does, to a certain degree, occur is seen in cases of spondylolisthesis, a condition which is far commoner than is usually thought. Mathematically, it can be shown that for an angle of 30 degrees the shearing force amounts to 43 per cent. of the superincumbent weight. The lumbo-sacral inclination lies a little above the centre of gravity in a standing person; therefore it can be estimated that if he weighs $140 \mathrm{lb}$. the shearing force will amount to about $27 \mathrm{lb}$; which is much smaller than one would have thought (Fig. 7 B). Obviously in carrying weights the force will increase proportionately.

In an intact last lumbar vertebra the interlocking of its lower articular facets with those of the sacrum clearly counterbalance shear. The ilio-lumbar ligaments play a part, as does also the intervertebral disc. I am going into this in more detail because I believe that in the human machine and its disabilities not enough attention is directed to the internal stressing of its materials - in this region as important as anywhere. Of particular importance is the nucleus pulposus which though composed of soft tissues expands when released from its coverings; thus constituted it is clearly compressible. If in a lumbo-sacral skeleton one places a steel ball of the same size and in the same position as the nucleus pulposus, but embed it in rubber instead of the annulus, and then erect the specimen in a position comparable to that of erect posture with a superimposed weight, interesting information can be obtained. Briefly the ball sets up reactionary forces (Newton's Law) the effect of which is to "push"

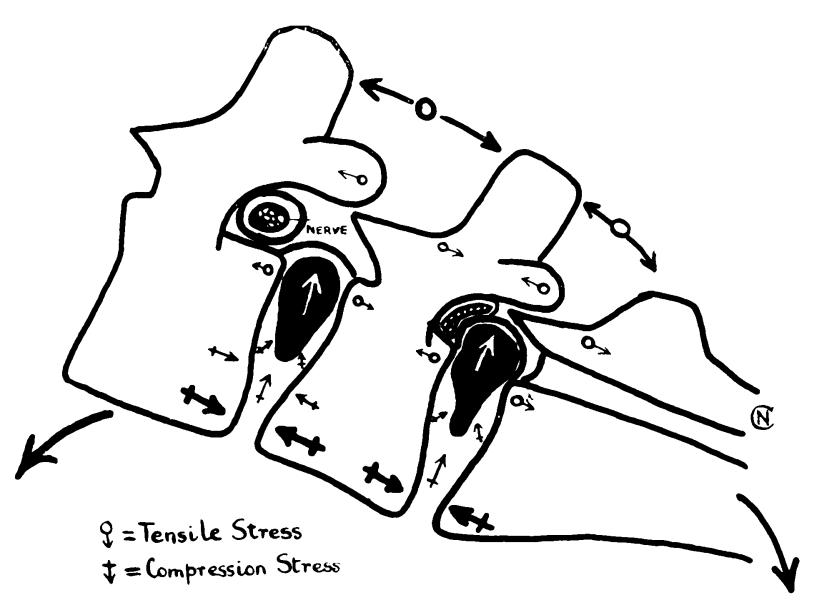

A

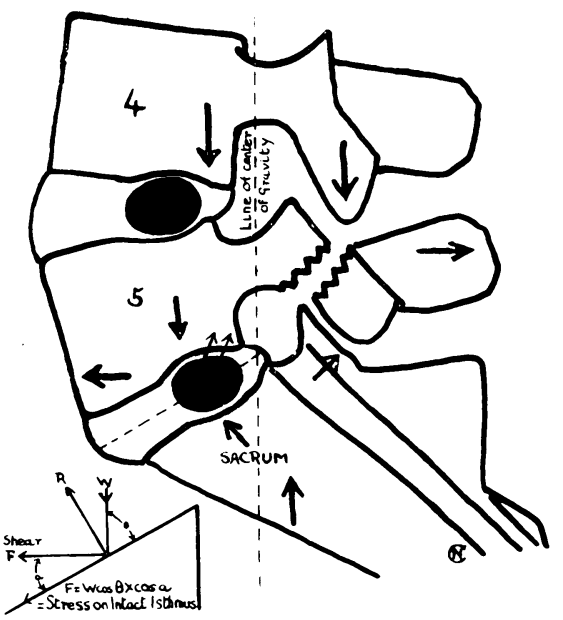

B

FIG. 7.-A.-The mechanics of nuclear retropulsion. B.-The mechanics of spondylolisthesis (the nucleus pulposus exerts reactionary force to shear). 
the last lumbar vertebra backwards and upwardsin other words the ball tends to prevent it from sliding forwards. In the body, however, as the nucleus is a compressible structure its influence mechanically is even greater than the ball, because under weight it exposes a larger surface for the opposition of shearing force. In spondylolisthesis, where there is lack of continuity in the posterior neural arch between the upper and lower articular facets, the influence of restraint by both the ilio-lumbar ligaments and by the nucleus pulposus is probably much greater. Spondylolisthesis in its earlier state, that is spondylolysis, is probably present in about 5 per cent. of people, a frequency that adds point to discussions of lumbo-sacral vulnerability.

\section{Lumbo-sacral Musculature}

In 1934, before we heard much about the lesions of the intervertebral discs, I stated that I belleved that 75 per cent. of the disturbance of lumbo-sacral sciatica was of a physiological nature-meaning muscle spasm. This may be an exaggeration; nevertheless I still believe it is near to the truth. The wrenched ligament, the retropulsed intervertebral nucleus, the stretched nerve root, whatever the inner lesion, is merely the trigger released; the explosion is in the muscular system. It may be large or small. What we later have to contend with is the atrophy and the dissociation of coordination in the muscles. Such atrophy, so obvious in the thigh muscles after derangement of the knee, is not so apparent in spinal lesions.

A simple picture of the posterior spinal musculature can be obtained if we regard it as comprising a continuous mass arising mainly from the sacrum and ilium on each side and passing upwards towards the neck, where it becomes more specialized. Throughout its course its deeper surface receives reinforcements from the bony structures upon which it lies. The superficial part of the mass splits into three perpendicular bundles attached respectively to the rib angles, the transverse and spinous processes. The deeper part of the muscle comprises shorter muscle units which have a more oblique course according to depth, but in the main they pass from transverse processes below, upwards and inwards to the spinous processes one or more vertebrae above, the more superficial passing over five or more vertebrae. The anterior spinal muscles are the abdominal muscles. The lateral spinal muscles comprise the extensions of the abdominal muscles into the loin, together with the psoas and a muscle which is often overlooked-namely, the quadratus lumborum. This arises from the iliac crest and iliolumbar ligament and passes upwards to be attached to the tips of the lumbar transverse processes and to the lowest rib. It is described as being a lateral flexor of the spine and a weak extensor. As a lateral flexor it is necessarily affected by the rotation (or torsion) that always accompanies side bending. The quadratus lumborum lies very close to the axis of backward and forward movement-so close, in fact, that in forward flexion it is possible for the muscle to act as a flexor. Normally spinal flexion rarely needs any flexor muscles; it is a gravity aided action which chiefly requires extensor muscles to control it. One can, without greatly stretching the imagination, appreciate circumstances in which, the "trigger" having gone off with the lumbar spine flexed, the quadratus lumborum is caught by explosive muscle spasm in a flexion phase. Should the spine also be twisted at the same time, it is possible for the quadratus on one side to be fixed in flexor spasm while the opposiie is in extension. This is somewhat hypothetical; but I believe that in some such disorder is to be found one of the explanations of what, to some patients with an acute attack of lumbago, is an almost catastrophic disturbance. These muscles must, therefore, for coordinated action require a nice adjustment of control. Under conditions of fatigue or strain, physical or mental, this regulation may more readily break down. Is it too fanciful to regard minor degrees of such disturbance in other muscles as well, by causing atrophy, fibrosis, and adhesions, to be the same thing as much of the fibrositis about which we hear in sciatica?

It is worth considering the phases of muscular activity which have to be coordinated; thus can the complexity of the spinal problem be more fully appreciated. These are :-

A. Simple Shortening Contraction.-The " normal" anatomical action, e.g. of the biceps in flexing the elbow or the gluteals in extending the pelvis on the femur.

B. Maintained Contraction (static).-As when the quadriceps is tensed without movement of the knee.

C. Lengthening Relaxation.-As when the triceps elongates to permit the biceps to flex the elbow.

D. Lengthening Contraction.-The converse to A and seen when a weight in the hand is lowered by extension of the elbow. This is the "paradoxical" action of the biceps. It is the same action that the spinal extensors have in controlling flexion of the spine and in the quadriceps particularly in walking down hill.

E. Maintained Relaxation.-The static condition of complete relaxation.

F. Shortening Relaxation.-The converse to C, as when the triceps shortens during the paradoxical action of the biceps in controlling elbow extension against gravity. Similarly in the spinal flexors when the extensors are in action against flexion of the spine.

\section{Summary}

The lumbar spine presents many features in anatomy and mechanics the appreciation of which should help in the elucidation of the clinical problems of sciatica. It remains to state that in clinical investigations more note should be taken of such factors.

The history should have regard for details of trauma, particularly of the more slowly operative or static types. The habitual postures of occupation 
should be inquired into as well as the posture assumed during any isolated incident which has precipitated an acute sciatic attack. Such an inquiry may be found just as important as the wellrecognized details of the mechanics of knee-cartilage injuries. Additional information should be sought about those postures which either aggravate or relieve symptoms-matters which will be available for corroboration during the physical examination. During the latter more attention than is usual should be paid to the influences of spinal movement upon the intraspinal and intervertebral tissues and to the changes in intrathecal pressure. Finally, we should make a closer study of the muscular control of the lumbar region.

In this paper I have presented information and a few theories about the mechanics of the lumbosacral region. Amongst the points I have discussed from a new angle are the rotation of the lumbar spine, the movements of the meninges and nerve roots, the mechanics of the nucleus pulposus, weight transmission and matters relating to the "internal stresses of materials," and the action of the quadratus lumborum muscles. In the course of the paper it has been necessary to touch on some very elementary facts, but the whole is given as a guide to the investigation and elucidation of the problems of sciatica for clinical workers.
The researches upon which this is a brief report started in the departments of anatomy, radiology, and orthopaedics at the University of Michigan and have been continued during the past twelve years in the orthopaedic hospitals of Devon. It owes much to the inspiration of the late Dr. P. M. Hickey, Professor of Roentgenology at the University of Michigan and to Sir Arthur Keith. To Professor F. H. Newman of the Department of Physics, University College of the South West, Exeter, I am most grateful for his guidance upon the mechanical problems involved. For the radiological studies upon normal spines in standing and other postures, I am indebted to the patience of various past and present members of my Staff in the Princess Elizabeth Orthopaedic Hospital, Exeter.

\section{REFERENCES}

Alajouanine, I., and Petit-Dutaillis, D. (1930). Presse Mèd., 38, 1657, 1749.

Beadle, O. A. (1931). M. R. C., Special Report No. 161.

Capener, N. (1932). Brit. J. Surg., 19, 374

(1933), Proc. roy. Soc. Med., 26, 29.

(1934). J. Chart. Soc. Mass. Med.Gym., Special Congress No.

- (1937). Proc. roy. Soc. Med., 30, 54.

Cunningham (1937). Textbook of Anatomy. Seventh Edition. Plate XVII, p. 328.

Danforth, M. S., and Wilson, P. D. (1925). J. Bone and Joint Surg., 7, 109.

Dockerty, M. B., and Love, J. G. (1940). Proc. Mayo Clin., 15, 161

Frazer, J. E. (1933). The Anatomy cf the Human Skeleton.

Lambrinudi, C. (1934). J. Bone and Joint Surg., 17, 239.

Mixter, W. J., and Barr, J. S. (1934). New England J. Med., 211, 210.

Peet, M. M., and Echols, D. H. (1934). Arch. Neurol. and Psych. Peet, 32. $_{924}$.,

Willis, J. A. (1932). J. Bone and Joint Surg., 14, 267. 